DOI: https://doi.org/10.46296/gt.v1i2.0003

\title{
DISEÑO DEL PROGRAMA DE FORMACIÓN EN MODALIDAD E- LEARNING PARA EL HOSPITAL MÓVIL \#1 DEL ECUADOR
}

\section{DESIGN OF THE TRAINING PROGRAM IN E-LEARNING MODE FOR THE MOBILE HOSPITAL \# 1 IN ECUADOR}

\author{
Durán-Solórzano Stéfano Alexander ${ }^{1 *}$ \\ Martínez-Minda Héctor Alfredo² \\ ${ }^{1}$ Escuela Superior Politécnica del Litoral, ESPOL. Guayaquil, Ecuador. \\ ${ }^{2}$ Escuela Superior Politécnica del Litoral, ESPOL. Guayaquil, Ecuador.
}

*Correo: sads.2050@gmail.com

\begin{abstract}
Resumen
La capacitación es un proceso de mejora continua, que utiliza la evaluación como elemento principal para retroalimentarse en las necesidades de la organización, además, se define como un medio para alcanzar las metas planteadas. En concordancia con lo mencionado, el objetivo de una propuesta basada en la capacitación virtual permite contribuir al mejoramiento de la atención a los pacientes que acuden al hospital móvil № 1, mediante la implementación del programa de formación en modalidad E-learning para optimizar los procesos. Con el compromiso y el propósito de mejorar los resultados en el desempeño de las labores del personal del Hospital Móvil № 1, se realizó un plan de capacitación que facilite el aprendizaje y desarrollo del personal de salud. Los resultados y aplicación de la plataforma posibilitaron en un tiempo corto, realizar los procesos de actualización. Por su parte, E-Learning, facilitó el desarrollo de clases que permitió potenciar el interés en las capacitaciones. Finalmente, para que la capacitación en línea genere resultados significativos, demanda de una fuerte dosis de compromiso continuo, que permita alcanzar la excelencia.
\end{abstract}

Palabras clave: Capacitación virtual, planificación, evaluación, hospital,

\begin{abstract}
Training is a process of continuous improvement, which uses evaluation as the main element to feed back into the needs of the organization, and it is also defined as a means to achieve the goals set. In accordance with the aforementioned, the objective of a proposal based on virtual training allows to contribute to the improvement of care for patients who come to mobile hospital No. 1, by implementing the training program in E-learning mode to optimize processes. With the commitment and purpose of improving the performance results of the work of the staff of the Mobile Hospital No. 1, a training plan was carried out to facilitate the learning and development of health personnel. The results and application of the platform made updating processes possible in a short time. For its part, E-Learning, facilitated the development of classes that allowed to enhance interest in training. Finally, in order for online training to generate significant results, it requires a strong dose of continuous commitment that enables excellence to be achieved.
\end{abstract}

Keywords: Management, quality, training, hospital, workers.

Información del manuscrito:

Fecha de recepción: 06 de abril de 2018

Fecha de aceptación: 14 de junio de 2018

Fecha de publicación: 10 de julio de 2018 


\section{Introducción}

La competitividad de las organizaciones de la salud exige el fortalecimiento e intervención efectiva y oportuna en los procesos de rutina de la atención primaria, por tal razón se deben proporcionar actualizaciones de manera permanente, tanto al personal de servicio, asistentes sociales, médicos y enfermeras, entre otros (Brito et al., 2017).

Actualmente se cuenta con los recursos tecnológicos que permiten acceder a capacitaciones por Internet, los entrenamientos pueden abarcar, desde los procesos de atención a los pacientes, hasta los procedimientos para hacer una intervención breve y referir a la persona para un tratamiento más extenso. Las capacitaciones en Modalidad E-learning, posibilitan la efectividad en la aplicación de procesos que permitan mejorar la calidad de servicio a los pacientes (Llewlyn, 2009).

Se evidencia también que no existe un canal de capacitación del talento humano de parte de instituciones hospitalarias (Cruz-Vega et al., 2016), además, se puede mencionar que incumplen con este aspecto, la
Cámara de Industrias de Guayaquil, este gremio aglutina a muchas empresas que desarrollan diferentes actividades y contribuyen al servicio de la comunidad y desarrollo productivo del país, en razón de que su aporte en diferentes ámbitos es significativo, como son el sector de la construcción, manufacturas, plásticos, farmacéuticas, alimentos, entre otras; por lo que necesitan estar en constante actualización de procesos, y procedimientos productivos, para minimizar costos y maximizar utilidades en la producción de diferentes áreas que son realizadas con el aporte del talento humano (Leibowicz, 2011).

Cabe destacar que el Hospital Móvil $\# 1$, es una unidad hospitalaria de gran apoyo logístico y operacional para el hospital público Abel Gilbert Pontón, de la ciudad de Guayaquil, por consiguiente, su personal e infraestructura tiene que estar eficientemente operativa, para dar un servicio que cubra los estándares de calidad y de esta manera proporcionar una asistencia integral de salud (MSP, 2008).

En base a lo mencionado previamente, el objetivo de una propuesta basada en la capacitación 
virtual es contribuir al mejoramiento de atención a los pacientes que acuden al hospital móvil № 1, mediante la implementación del programa de formación en modalidad E-learning, para optimizar los procesos.

\section{Metodología}

La aplicación de las TIC ha facilitado el proceso socio-comunicativo, actualmente ofrece grandes ventajas para la adquisición de conocimientos, ante la gran demanda de usuarios, los entornos virtuales han ido en aumento, pues son muchas las ventajas que estos ofrecen, para lograr capacitación y actualización, entre las que se pueden mencionar las siguientes: Fácil adaptación continua, están adecuadas a las necesidades del individuo, se las puede usar de acuerdo a la disponibilidad del tiempo del usuario, rápida actualización (Demirdjian et al., 2017)

\subsection{Arquitectura}

El modelo de arquitectura que se presenta, introduce una concepción modular de aplicaciones web para ELearning, en este caso ha sido implementado, bajo componentes de la Plataforma Academia Experta. El esquema permite en un futuro realizar aplicaciones que permitan al usuario proporcionar diferentes tipos de capacitaciones. En la figura 1 se muestra un modelo de arquitectura para el sistema E-Learning.

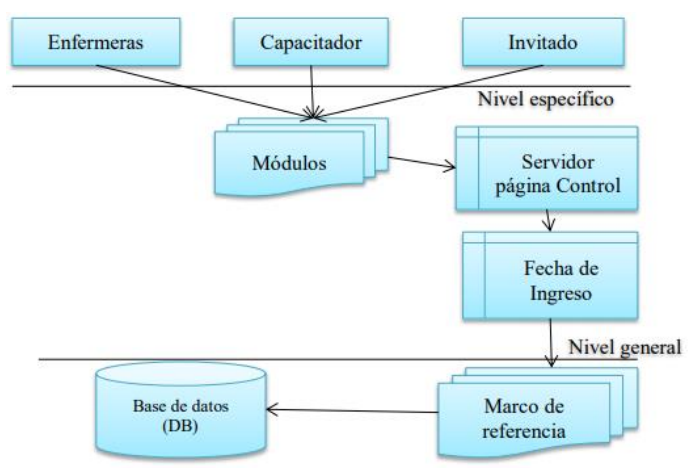

Figura 1. Modelo de Arquitectura del sistema E-Learning: lavado de manos

\subsection{Plan del curso}

Con el compromiso y el propósito de mejorar los resultados en el desempeño de las labores del personal del Hospital Móvil № 1, se debe previamente realizar un plan de capacitación que facilite el aprendizaje y desarrollo del personal de salud.

Previamente, se tomaron las decisiones coherentes a las necesidades de aprendizaje $y$ actualizaciones, para determinar el enfoque adecuado con el perfil del participante, en razón de que unas de las limitantes que afectan la 
eficacia de las capacitaciones es la ausencia del análisis previo del problema o necesidad que se espera mejorar, mediante el uso de los recursos tecnológicos, cuya utilización debe ser direccionada por técnicos informáticos 0 docentes tutores, cabe indicar que la operatividad es sencilla, por lo que brinda la posibilidad de ser utilizada por todos los profesionales.

\section{Resultados y discusión}

\subsection{Descripción del contenido}

En el presente caso se seleccionaron los contenidos en módulos temáticos como a continuación se describen.

Tabla 1. Módulo 1: Proceso de atención de consulta externa

\begin{tabular}{|l|c|c|}
\hline \multicolumn{1}{|c|}{ Temática } & Tiempo & \multirow{2}{*}{ Total de Horas } \\
\cline { 1 - 2 } Motivación para el cambio & 30 minutos & \\
\cline { 1 - 2 } Orientación adecuada al paciente & 30 minutos & \multirow{2}{*}{ 2 horas y media } \\
\cline { 1 - 2 } Registro diario de pacientes de Consulta externa & 30 minutos & \\
\cline { 1 - 2 } Expediente clínico & 30 minutos & \\
\cline { 1 - 2 } Registro de citas medicas & 30 minutos & \\
\hline
\end{tabular}

Tabla 2. Módulo 2: Proceso para seguimiento-Emergencias

\begin{tabular}{|l|c|c|}
\hline \multicolumn{1}{|c|}{ Temática } & Tiempo & Total de Horas \\
\cline { 1 - 2 } Atención masiva de pacientes en caso de desastres & 30 minutos & \\
\cline { 1 - 2 } $\begin{array}{l}\text { Atención de pacientes que presentan urgencias } \\
\text { médico quirúrgicas }\end{array}$ & 30 minutos & \multirow{2}{*}{2 horas } \\
\cline { 1 - 2 } Tipos de urgencia en las unidades de emergencia & 30 minutos & \\
\cline { 1 - 2 } Actividades en la unidad de emergencia & 30 minutos & \\
\hline
\end{tabular}

Tabla 3. Módulo 3: Seguimiento de lavado de manos

\begin{tabular}{|l|r|r|}
\cline { 1 - 2 } \multicolumn{1}{|c|}{ Temática } & Tiempo & Total de Horas \\
\cline { 1 - 2 } Lavado de manos & 15 minutos & \\
\cline { 1 - 2 } Tipo de lavado de manos & 15 minutos & \multirow{2}{*}{1 horas } \\
\cline { 1 - 2 } Recomendaciones para higiene de manos & 15 minutos & \\
\cline { 1 - 2 } Secuencia de un correcto lavado de manos & 15 minutos & \\
\hline
\end{tabular}

\subsection{Implementación}

La implementación de la capacitación virtual podría implicar el mejoramiento continuo de una empresa, por tal razón debe ser implementada, de esta manera se pueden solucionar las dificultades que se presentan en el campo laboral, y así alcanzar la competitividad. En este caso fue seleccionada la Plataforma de la Academia Experta, que pertenece a un grupo de empresas capacitadoras a nivel nacional.

Este diseño de sistemas basados en tecnología e-learning, es desarrollado mediante componentes comunicativos con la creación de aulas virtuales, con servicio de diapositivas en línea, también, permiten transmitir de forma eficiente teleclases y vídeos, que de una forma u otra ayudan al estudiante 0 profesional, no sólo a enfrentarse al sistema, sino a utilizar estos elementos tecnológicos para 
resolver problemas (Cruz-Vega et al., 2016).

\subsection{Aplicación}

Los resultados y aplicación de la plataforma posibilitaron en un tiempo corto, realizar los procesos de actualización. Además, E-Learning, facilitó el desarrollo de clases que permitió potenciar el interés en las capacitaciones (Vargas et al., 2017). Cabe resaltar que este sistema es usado por miles de usuarios, los estudiantes y empresas comerciales juegan un papel protagonista, los cuales han emitido criterios favorables acerca de este sistema (Avello-Martínez \& Duart, 2016; Mejía \& López, 2016).

A lo largo de los planteamientos efectuados, es evidente que este estudio incursionó en las capacitaciones a distancia en modalidad virtual, como alternativa para lograr un mayor alcance en los cursos de actualizaciones, dirigida a los integrantes del Hospital Móvil № 1. Para su implementación fue necesario en primer lugar seleccionar la plataforma con sistemas de E-learning más idónea, como es la Plataforma de la Academia Experta, que permite ofrecer la solución adecuada para la propuesta planteada.

\subsection{Elementos para la gestión de la capacitación}

Sin duda alguna el desarrollo del personal en las instituciones debe integrarse con todas las funciones del sistema. Por tal razón la capacitación y actualización deben ser permanentes para mejorar el desempeño de las personas. Los componentes básicos se encuentran descritos en la figura 2.

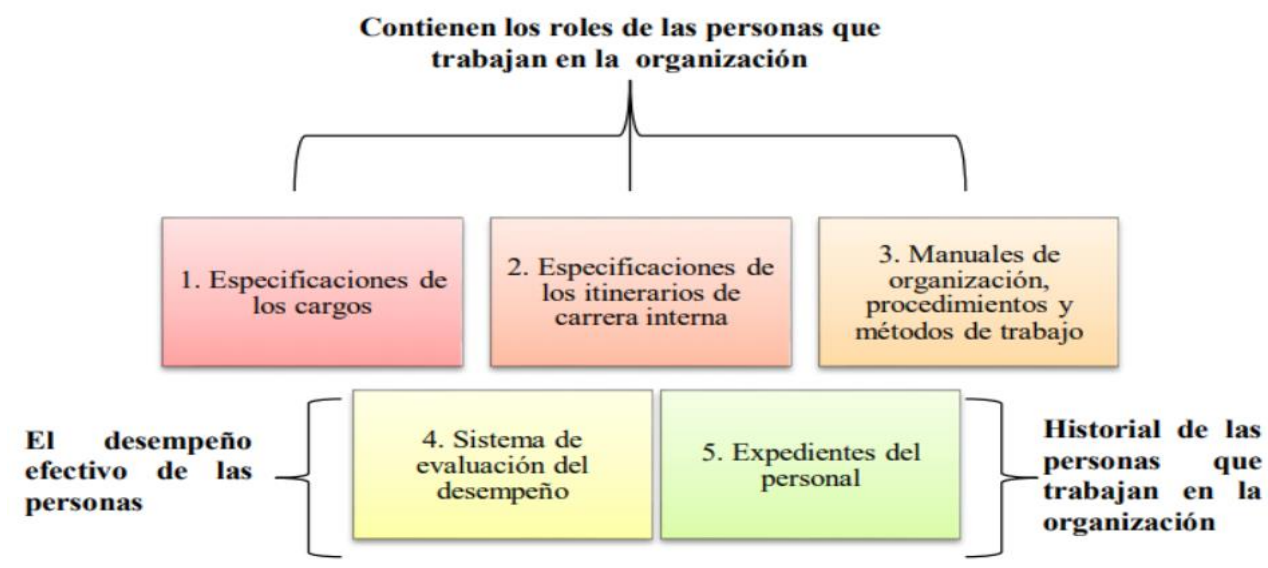

Figura 2. Elementos básicos para la gestión de la capacitación 
Para realizar una buena gestión de capacitación, es necesario establecer los siguientes procesos fundamentales en la formación, por lo que se deben cumplir las diferentes etapas expuestas en la figura 3.

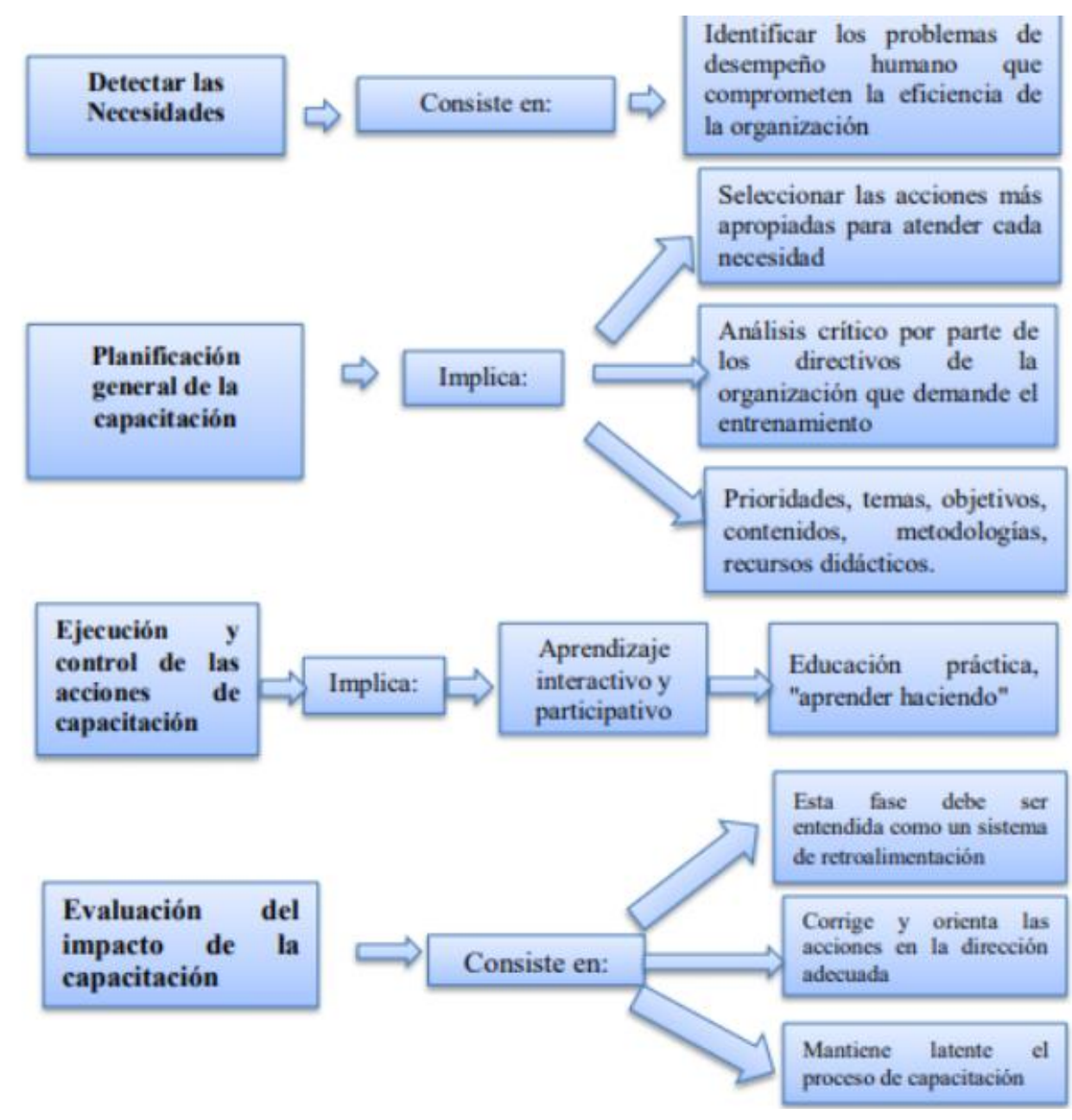

Figura 3. Etapas de la gestión de la capacitación

\subsection{Proceso de desarrollo}

El proceso de desarrollo consta de varias actividades que a continuación se describen:

a. Compilación de información: es la recolección de información requerida. Esta actividad se realiza con la aplicación de entrevistas, encuestas u observación.

b. Análisis: es el estudio de la información recopilada, que permite identificar los esquemas comunes.

c. Modelamiento: aporta el detalle necesario que permite su entendimiento. 
d. Síntesis: constituye en una evaluación del modelo generado, identificando los elementos clave que puedan aportar antecedentes para la toma de decisiones en torno a la institución y a la habilitación de las TIC.

\subsection{Criterios para la oferta de capacitación en línea}

Para que la capacitación en línea genere resultados significativos, demanda de una fuerte dosis de compromiso continuo, que permita alcanzar la excelencia. Este inicia con el diseño del módulo, continúa con la elaboración de la programación de los cursos en línea y se mantiene en los diferentes procesos que conlleva el desarrollo del entorno virtual (Salgado-Cruz et al., 2017).

Cabe señalar que las capacitaciones en línea demandan un cambio en la acción pedagógica y en la interacción e interactividad que se debe propiciar. Algunos de los criterios que deben ser considerados, para favorecer la búsqueda de la calidad de enseñanzas en línea, corresponden al diseño cuidadoso del módulo.
Luego de valorar los recursos, se debe realizar la matriz de programación, para establecer el tiempo, revisar, seleccionar y preparar las actividades, de igual manera, se recomienda, aplicar tablas de evaluación. Es imprescindible preparar el texto de bienvenida, las lecturas digitalizadas, los instrumentos de evaluación utilizados en las actividades, las direcciones URL que los vincule con la plataforma de la Academia Experta.

\section{Conclusiones}

Para establecer un programa de capacitación al personal de enfermería y las diferentes áreas de un Hospital Móvil, que permita mejorar los procesos en la atención, fue necesario apoyarse en la investigación de campo, que consecuentemente permitió concluir que existen puntos críticos que afectan el rendimiento del personal, por lo que es necesario implementar el plan de capacitación o actualización propuesto.

\section{Bibliografía}

Avello-Martínez, R., \& Duart, J. M. (2016). Nuevas tendencias de aprendizaje colaborativo en elearning: Claves para su 
implementación efectiva. Estudios pedagógicos (Valdivia), 42(1), 271-282.

Brito, L. A. L., Malik, A. M., Brito, E., Bulgacov, S., \& Andreassi, T. (2017). Prácticas de gestión en hospitales privados de tamaño medio de São Paulo, Brasil. Cadernos de Saúde Pública, 33(3).

Cruz-Vega, F., Loría-Castellanos, J., Hernández-Olivas, I. P., Franco-Bey, R., Ochoa-Avila, C., \& Sánchez-Badillo, V. (2016). Experiencia en capacitación en emergencias de la División de Proyectos Especiales en Salud, Instituto Mexicano del Seguro Social. Cirugía y Cirujanos, 84(2), 127-134.

Demirdjian, G., Rodríguez, S., Vassallo, J. C., Irazola, V., \& Rodríguez, J. (2017). Capacitación hospitalaria de profesionales pediátricos en investigación y gestión. Archivos argentinos de pediatría, 115(1), 58-64.

Leibowicz, J. (2011). Estrategias de Capacitación Basadas en TIC para Mipyme. p. 5. CINTERFOR.
Llewlyn, D. (2009). Planificación y Administración de Hospitales. En D. Llewlyn, Planificación y Administración de Hospitales (pág. 223). Londres: Publicación Científica No. 191.

Mejía, J. F., \& López, D. (2016). Modelo de Calidad de Elearning para Instituciones de Educación Superior en Colombia. Formación universitaria, 9(2), 59-72.

Ministerio de Salud Pública del Ecuador. (2008). Sumario: Función Ejecutiva. Acuerdo: Ministerio de Salud Pública: Quito. Registro Oficial № 151.

Salgado-Cruz, M., Gómez-Figueroa, O., \& Juan-Carvajal, D. T. (2017). Niveles para la capacitación en una organización. Ingeniería Industrial, 38(2), 154-160.

Vargas, P. R., Cano, C. A., \& Gil, J. M. S. (2017). Desde la educación a distancia al eLearning: emergencia, evolución y consolidación. Revista educación y tecnología, (10), 1-13. 